Discrete Comput Geom 33:207-221 (2005)

DOI: $10.1007 / \mathrm{s} 00454-004-1102-\mathrm{x}$

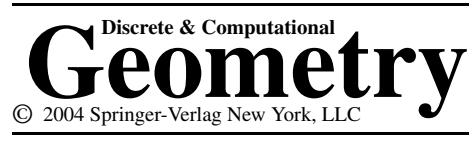

\title{
A Rigidity Criterion for Non-Convex Polyhedra
}

\author{
Jean-Marc Schlenker \\ Laboratoire Emile Picard, UMR CNRS 5580, Université Paul Sabatier, \\ 118 route de Narbonne, 31062 Toulouse Cedex 4, France \\ schlenker@picard.ups-tlse.fr \\ http://picard.ups-tlse.fr/ $/$ schlenker
}

\begin{abstract}
Let $P$ be a (non-necessarily convex) embedded polyhedron in $\mathbb{R}^{3}$, with its vertices on the boundary of an ellipsoid. Suppose that the interior of $P$ can be decomposed into convex polytopes without adding any vertex. Then $P$ is infinitesimally rigid. More generally, let $P$ be a polyhedron bounding a domain which is the union of polytopes $C_{1}, \ldots, C_{n}$ with disjoint interiors, whose vertices are the vertices of $P$. Suppose that there exists an ellipsoid which contains no vertex of $P$ but intersects all the edges of the $C_{i}$. Then $P$ is infinitesimally rigid. The proof is based on some geometric properties of hyperideal hyperbolic polyhedra.
\end{abstract}

\section{Introduction}

We are interested here in the infinitesimal rigidity of non-convex, embedded polyhedra in Euclidean 3-space, homeomorphic to the sphere. So we define polyhedra in the following way.

Definition 0.1. Let $P \subset \mathbb{R}^{3} . P$ is a polyhedron if there exists a finite triangulation $\tau$ of $S^{2}$ and a continuous, injective map $\varphi: S^{2} \rightarrow \mathbb{R}^{3}$, sending each face of $\tau$ to a triangle in a plane in $\mathbb{R}^{3}$, whose image is $P$.

There is then a natural notion of face of $P$ (the image by $\varphi$ of one of the faces of $\tau$ ), of edge of $P$ (the image of an edge of $\tau$ ) and of vertex of $P$ (the endpoints of the edges). Moreover, $P$ bounds a compact domain, which we call the interior of $P$. Note that it is not really necessary to suppose, in the above definition, that the faces of $P$ are triangles. For the infinitesimal rigidity questions, however, the general case is a direct consequence of the case where the faces are triangles. 
Each edge $e$ is in the boundary of two faces, and the dihedral angle of $P$ at $e$ is the angle between those faces, measured in the interior of $P$.

It is customary to say that $P$ is rigid if $P$ has no non-trivial one-parameter deformation among polyhedra with the same combinatorics and the same induced metrics (or edge lengths), otherwise it is flexible. We say that $P$ is infinitesimally rigid if any firstorder deformation of $P$ which does not change its combinatorics or its edges lengths is trivial-i.e. it is the restriction to $P$ of an infinitesimal isometry of $\mathbb{R}^{3}$. It is known that any infinitesimally rigid polyhedron is rigid, but the converse is false, see, e.g., [Co2].

The rigidity of polyhedra has a long history. Legendre [Le] and Cauchy $[\mathrm{Ca}]^{1}$ proved that convex polyhedra are rigid, and their proof can also be used to obtain that those polyhedra are infinitesimally rigid (a result first obtained by Dehn [De] by other methods). Their method has been extended by Stoker [St] and more recently by Rodríguez and Rosenberg $[\mathrm{RR}]$ to some non-convex polyhedra sharing some properties of convex polyhedra.

Bricard [Bri] introduced families of flexible octahedra, but they were not embedded, and thus not polyhedra according to the definition given above. Indeed it was conjectured for a long time that embedded polyhedra are rigid, until Connelly [Co1] found a counterexample. On the other hand, Sabitov [Sab] recently proved the "Bellows conjecture": the volume bounded by a flexible polyhedron remains constant.

The main goal of this paper is to prove an infinitesimal rigidity statement for some polyhedra. The proof is entirely different from the Legendre-Cauchy proof, and relies on hyperbolic geometry.

Theorem A. Let $P \subset \mathbb{R}^{3}$ be a polyhedron whose vertices are on the boundary of an ellipsoid. Suppose that the interior of $P$ can be decomposed as a union of convex polytopes, with disjoint interiors, without adding any vertex. Then $P$ is infinitesimally rigid.

It is quite easy to find examples of polyhedra to which this theorem applies; given at least five points in general position on a sphere, there are several polyhedra with those points as vertices. Our proof actually applies to a much larger class of polyhedra, for instance those obtained by moving the vertices of the examples of Theorem A a little. This is seen in the next theorem, which is more complicated but much more general than Theorem A.

Theorem A does not hold without the hypothesis on a decomposition into convex polytopes; there is a known example of an octahedron in $\mathbb{R}^{3}$ which has its vertices on a sphere but is not infinitesimally rigid.

Definition 0.2. Let $P$ be a Euclidean polyhedron, bounding a compact domain $\Omega$. A cellulation of $P$ is a decomposition of $\Omega$ into the union of a finite number of nondegenerate convex polytopes with disjoint interiors, such that the vertices of the $C_{i}$ are

\footnotetext{
${ }^{1}$ The fact that it was mostly due to Legendre was recently discovered by I. Sabitov.
} 

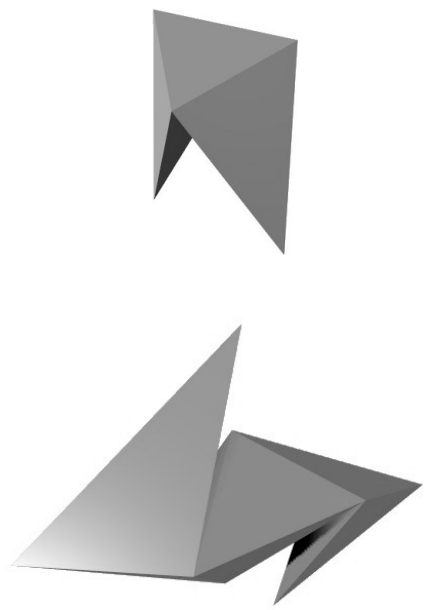
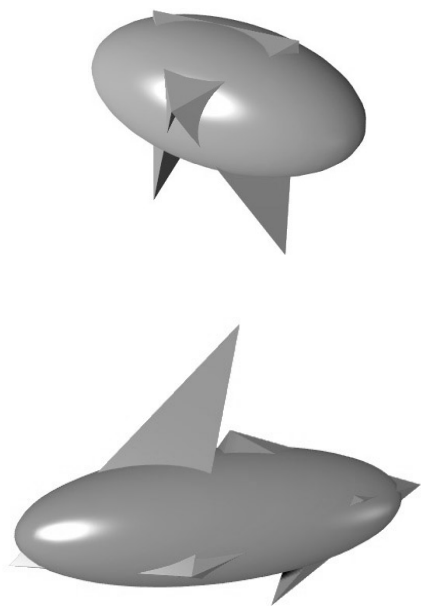

Fig. 1. Examples of infinitesimally rigid polyhedra.

vertices of $P$ and that, for $1 \leq i, j \leq n, C_{i} \cap C_{j}$ is a vertex, an edge or a face of $C_{i}$ and of $C_{j}$.

Theorem B. Let $P$ be a polyhedron, with a cellulation with cells $C_{1}, \ldots, C_{n}$. Let $E$ be an ellipsoid which contains no vertex of $P$, but intersects all edges of the $C_{i}$. Then $P$ is infinitesimally rigid.

Again it is easy to find polyhedra to which Theorem B applies. Some examples are shown in Fig. 1, along with the ellipsoid which shows that they are infinitesimally rigid.

Theorem A is a direct consequence of Theorem B: given a polyhedron $P$ with vertices on the boundary of an ellipsoid $E$, one can take a slightly smaller ellipsoid $E^{\prime}$ which contains no vertex of $P$, but intersects all the segments between two of those vertices. One can then apply Theorem B to obtain Theorem A.

The proof of Theorem B is based on hyperbolic geometry, and on related rigidity results for (non-convex) hyperideal polyhedra. Those objects are most easily described using the projective model of $H^{3}$, which identifies $H^{3}$ with the open unit ball $B_{0}$ in $\mathbb{R}^{3}$, and sends hyperbolic geodesics to segments in $B_{0}$; a hyperideal polyhedron is then the intersection with $H^{3}$ of a polyhedron which has all its vertices outside $\overline{B_{0}}$ but all its edges intersecting $B_{0}$. (Some definitions would allow the vertices to be on the boundary of $B_{0}$, but it would rather cloud the issues here.)

Given a hyperideal polyhedron, each vertex $v$ has a "dual plane", which is the hyperbolic plane which intersects orthogonally (for the hyperbolic metric) all the lines containing $v$. The length of an edge $e$ of $P$ is the hyperbolic distance, along $e$, between the planes dual to its endpoints; we sometimes call it the hyperbolic length of $e$, when there can be an ambiguity with the Euclidean length in the projective model. The dihedral angle of $P$ at an edge is also the angle for the hyperbolic metric. 
Definition 0.3. Let $P$ be a hyperideal polyhedron, bounding a compact domain $\Omega$ in the projective model. A hyperideal cellulation of $P$ is a decomposition of $\Omega$ as the union of a finite set of convex hyperideal polyhedra $C_{1}, \ldots, C_{n}$ with disjoint interiors, such that, for all $1 \leq i, j \leq n, C_{i} \cap C_{j}$ is a vertex, an edge or a face of $C_{i}$ and of $C_{j}$.

Theorem C. Let $P$ be a hyperideal polyhedron. Suppose that $P$ admits a hyperideal cellulation by polyhedra $C_{1}, \ldots, C_{n}$. Then any non-trivial first-order deformation of $P$ induces a non-trivial first-order variation of the hyperbolic lengths of its edges.

Theorem B will follow from Theorem C using the Pogorelov transformation, a remarkable tool which is recalled in Section 1. Along the way we find another infinitesimal rigidity statement for hyperideal polyhedra, concerning their dihedral angles.

Theorem D. Let $P$ be a hyperideal polyhedron, with a hyperideal cellulation by polyhedra $C_{1}, \ldots, C_{n}$. Any non-trivial first-order deformation of $P$ induces a non-trivial first-order variation of its dihedral angles.

Given a hyperideal polyhedron $P$, there is a compact polyhedron $P^{\prime}$, called the truncated polyhedron associated to $P$, which is obtained by truncating a neighborhood of each hyperideal vertex of $P$ by the hyperbolic plane dual to this vertex. A basic remark is that if $v, v^{\prime}$ are two points of $S_{1,+}^{3}$ such that the segment going from $v$ to $v^{\prime}$ (in the projective model of $S_{1,+}^{3}$ ) enters $B^{3}$, then the hyperbolic planes dual to $v$ and $v^{\prime}$ are disjoint. As a consequence, the combinatorics of $P^{\prime}$ is obtained from the combinatorics of $P$ by adding one face for each vertex of $P$. By construction, all the edges of those "new" faces have right angles, and all their vertices are trivalent (see [BB] for more details).

A consequence of Theorem $\mathrm{D}$ and of the Euler formula is that hyperideal polyhedra with given combinatorics, near one that satisfies the hypothesis of Theorem D, are parametrized by their dihedral angles. We can consider their volume, defined as the hyperbolic volume of the corresponding truncated polyhedron.

Theorem E. Let $P$ be a hyperideal polyhedron satisfying the hypothesis of Theorem $D$. In the neighborhood of $P$, the hyperideal polyhedra with the same combinatorics are parametrized by their dihedral angles. With this parametrization, their volume is a strictly concave function.

One of the underlying ideas of this paper, leading in particular to the proof of Theorem $\mathrm{D}$, is related to the methods developed to study circle packings and ideal polyhedra, in particular by Thurston [Th], Colin de Verdière [CdV], Brägger [Brä] and Rivin [Ri]. Thurston found the important relationship between the ideal polyhedra and the circle packings, and realized that the Koebe Circle Packing Theorem was a consequence of the Andreev Theorem on ideal polyhedra [An]. He also gave an extension of the Koebe Theorem (to hyperbolic surfaces, etc.) and a proof which apparently helped Colin de Verdière discover that there was a variational approach to those results. Brägger discovered that the underlying functional was the hyperbolic volume. Rivin rediscovered this independently, refined and simplified the proof, and brought it from the setting of circle 
packings to ideal polyhedra; the path that we follow in Section 3 is directly related to his paper [Ri]. This approach was first transfered to the setting of hyperideal polyhedra in [Sc4].

We remark that it is not really necessary to suppose that the polyhedra we consider are embedded. It is necessary to have them immersed, but they might bound a domain which includes some parts which are "multiply covered".

The proof of Theorem B could be done entirely in the setting of Euclidean geometry; hyperbolic geometry enters the picture only through the hyperbolic volume functional and its wonderful properties. This suggests that other functionals could be used to prove infinitesimal rigidity results. One could for instance wonder whether any polyhedron with vertices on a strictly convex sphere in $\mathbb{R}^{3}$, which can be decomposed as a union of polytopes with disjoint interiors with no new vertex, is infinitesimally rigid.

\section{The Pogorelov Transformation}

We recall in this section some fairly well-known facts of hyperbolic geometry, in particular the Pogorelov transformation, which takes infinitesimal rigidity questions from $H^{3}$ or $S_{1}^{3}$ to Euclidean 3-space. This transformation was defined by Pogorelov [Po] in the case of $H^{3}$ and $S^{3}$.

The three-dimensional de Sitter space is a Lorentzian space of constant curvature 1. Like $H^{3}$, it can be obtained as a quadric in the Minkowski four-dimensional space $\mathbb{R}_{1}^{4}$. Recall that $\mathbb{R}_{1}^{4}$ is simply $\mathbb{R}^{4}$, with the Lorentz metric:

$$
d x^{2}+d y^{2}+d z^{2}-d t^{2} .
$$

The de Sitter space, like $H^{3}$, can be seen as a quadric in $\mathbb{R}_{1}^{4}$, with the induced metric:

$$
S_{1}^{3}:=\left\{x \in \mathbb{R}_{1}^{4} \mid\langle x, x\rangle=1\right\} .
$$

We call $S_{1,+}^{3}$ the "positive hemisphere" defined, in the hyperboloid model, as the set of points of $S_{1}^{3}$ with positive vertical coordinate $t$. There is a "projective model" of $S_{1,+}^{3}$. It is a map $\varphi_{S}: S_{1,+}^{3} \rightarrow \mathbb{R}^{3} \backslash \overline{B_{0}}$, which is defined by sending a point $x \in S_{1,+}^{3}$ in the hyperboloid model to the intersection of the line containing 0 and $x$ with the plane $\{t=1\}$, where $t$ is the vertical coordinate.

Each totally geodesic, space-like plane $P_{0}$ of $S_{1}^{3}$ bounds two "hemispheres" each isometric to $S_{1,+}^{3}$. We will fix a plane $P_{0}$, which in the hyperboloid model will be the intersection of $S_{1}^{3}$ with the hyperplane $\{t=0\}$. The time-like geodesics orthogonal to $P_{0}$ foliate $S_{1}^{3}$. At each point $x \in S_{1}^{3}$, we call the directions which are parallel to the unique geodesic containing $x$ and orthogonal to $P_{0}$ "radial", and the directions which are orthogonal to this direction "lateral". By construction, the differential $d \varphi_{S}$ of $\varphi_{S}$ sends the radial direction in $S_{1,+}^{3}$ to the radial direction in $\mathbb{R}^{3}$, and the lateral directions in $S_{1,+}^{3}$ to the lateral directions in $\mathbb{R}^{3}$.

Also, the oriented distance from $x$ to $P_{0}$ along the time-like geodesic containing $x$ and orthogonal to $P_{0}$ we call $\rho$. The spheres $\left\{\rho=\rho_{0}\right\}$, for $\rho_{0} \in \mathbb{R}$, are totally umbilical, space-like spheres. 
Definition 1.1. We define $\Phi_{S}: T S_{1,+}^{3} \rightarrow T\left(\mathbb{R}^{3} \backslash \overline{B_{0}}\right)$ as the map sending $(x, v) \in T S_{1,+}^{3}$ to $\left(\varphi_{S}(x), w\right)$, where:

- The lateral component of $w$ is the image by $d \varphi_{S}$ of the lateral component of $v$.

- The radial component of $w$ has the same direction and the same norm as the radial component of $v$.

The following remark is at the heart of the proof of Lemma 1.3 below.

Remark 1.2. For each $\rho \in \mathbb{R}_{+}$, call $I_{\rho}$ and $I_{\rho}$ the induced metric and second fundamental form of the sphere at distance $\rho$ from $P_{0}$ in $S_{1}^{3}$; for each $t \in(1, \infty)$, call $\bar{I}_{t}$ and $\overline{I I}_{t}$ the induced metric and second fundamental form of the sphere of radius $t$ in $\mathbb{R}^{3}$. Then, for $t=1 / \tanh (\rho)$, we have

$$
I_{\rho}=\sinh ^{2}(\rho) \bar{I}_{t}, \quad I_{\rho}=\sinh ^{2}(\rho) \overline{I I}_{t} .
$$

Proof. Let "can" be the canonical metric on $S^{2}$, then $I_{\rho}=\cosh ^{2}(\rho)$ can, $I_{\rho}=$ $\sinh (\rho) \cosh (\rho) \operatorname{can}, \bar{I}_{t}=t^{2}$ can, $\bar{I}_{t}=t$ can; the result follows.

We can now state the main property of the Pogorelov transformation. The next lemma is basically taken from [Sc3], but we include the proof since [Sc3] contains only the proof of the analog for $H^{3}$ instead of $S_{1,+}^{3}$.

Lemma 1.3. Let $S$ be a smooth submanifold in $S_{1,+}^{3}$, and let $v$ be a vector field of $S_{1,+}^{3}$ defined on $S$; then $v$ is an isometric deformation of $S$ if and only if $\Phi_{S}(v)$ is an isometric deformation of $\varphi_{S}(S)$. In particular, if $v$ is a vector field on $S_{1,+}^{3}$, then $v$ is a Killing field if and only if $\Phi_{S}(v)$ is a Killing field of $\mathbb{R}^{3}$.

Proof. The second part follows from the first by taking $S=S_{1,+}^{3}$.

To prove the first part, we have to check that the Lie derivative of the induced metric on $S$ under $v$ vanishes if and only if the Lie derivative of the induced metric on $\varphi_{S}(S)$ under $\Phi_{S}(v)$ vanishes. In other words, if $x, y$ are vector fields tangent to $S$, if we call $g$ and $\bar{g}$ the metrics on $S_{1,+}^{3}$ and $\mathbb{R}^{3}$, respectively, and $\bar{x}=d \varphi_{S}(x), \bar{y}=d \varphi_{S}(y)$, then we have to prove that

$$
\left(\mathcal{L}_{v} g\right)(x, y)=0 \quad \Leftrightarrow \quad\left(\mathcal{L}_{\Phi_{S}(v)} \bar{g}\right)(\bar{x}, \bar{y})=0 .
$$

We will prove that the two terms are actually proportional:

$$
\left(\mathcal{L}_{v} g\right)(x, y)=\sinh ^{2}(\rho)\left(\mathcal{L}_{\Phi_{S}(v)} \bar{g}\right)(\bar{x}, \bar{y}) .
$$

We decompose $v$ into the radial component $f N$, where $N$ is the unit radial vector, and the lateral component $u$. Then $\Phi_{S}(v)=f \bar{N}+\bar{u}$, where $\bar{N}$ is the unit radial vector in $\mathbb{R}^{3}$, and $\bar{u}:=d \varphi_{S}(u)$.

By linearity, it is sufficient to prove (1) in the cases where $x$ and $y$ are non-zero, and are each either radial or lateral. We consider each case separately, and call $D$ and $\bar{D}$ the Levi-Cività connections of $S_{1,+}^{3}$ and $\mathbb{R}^{3}$, respectively. 
First case: $x$ and $y$ are both radial. Then

$$
\left(\mathcal{L}_{v} g\right)(x, y)=\left(\mathcal{L}_{u} g\right)(x, y)+\left(\mathcal{L}_{f N} g\right)(x, y) .
$$

However,

$$
\left(\mathcal{L}_{u} g\right)(x, y)=g\left(D_{x} u, y\right)+g\left(x, D_{y} u\right)=0,
$$

because both $D_{x} u$ and $D_{y} u$ are lateral. Moreover,

$$
\left(\mathcal{L}_{f N} g\right)(x, y)=g\left(D_{x} f N, y\right)+g\left(x, D_{y} f N\right)=d f(x) g(N, y)+d f(y) g(x, N) .
$$

The same computation applies in $\mathbb{R}^{3}$. In addition, $d f(x)=d f(\bar{x})$ by definition of $\bar{x}$, so the scaling comes only from $g(x, N)$ versus $\bar{g}(\bar{x}, N)$. So (1) is true in this case.

Second case: $x$ and $y$ are both lateral. Then, at a point at distance $\rho$ from $x_{0}$, with $t:=1 / \tanh (\rho)$, we have by Remark 1.2 that

$$
\left(\mathcal{L}_{u} g\right)(x, y)=\left(\mathcal{L}_{u} I_{\rho}\right)(x, y)=\sinh ^{2}(\rho)\left(\mathcal{L}_{\bar{u}} \bar{I}_{t}\right)(\bar{x}, \bar{y})=\sinh ^{2}(\rho)\left(\mathcal{L}_{\bar{u}} \bar{g}\right)(\bar{x}, \bar{y}) .
$$

Moreover,

$$
\left(\mathcal{L}_{f N} g\right)(x, y)=g\left(D_{x} f N, y\right)+g\left(x, D_{y} f N\right)=-2 f I_{\rho}(x, y),
$$

and since the same computation applies in $\mathbb{R}^{3}$, we see by Remark 1.2 that

$$
\left(\mathcal{L}_{f N} g\right)(x, y)=\sinh ^{2}(\rho)\left(\mathcal{L}_{f N} \bar{g}\right)(\bar{x}, \bar{y})
$$

and

$$
\left(\mathcal{L}_{v} g\right)(x, y)=\sinh ^{2}(\rho)\left(\mathcal{L}_{\bar{v}} \bar{g}\right)(\bar{x}, \bar{y}),
$$

so that (1) also holds in this case.

Third case: $x$ is lateral, while $y$ is radial. We choose an arbitrary extension of $x$ and $y$ as vector fields which remain tangent, resp. orthogonal, to the spheres $\{\rho=$ const $\}$. Then

$$
\left(\mathcal{L}_{u} g\right)(x, y)=u \cdot g(x, y)-g([u, x], y)-g(x,[u, y])=-g(x,[u, y]),
$$

because $[u, x]$ is tangent to the sphere of radius $\rho$. So, since the Lie bracket does not depend on the metric, we have by Remark 1.2 that

$$
\left(\mathcal{L}_{u} g\right)(x, y)=-g(x,[u, y])=-\sinh ^{2}(\rho) \bar{g}(x,[u, y])=\sinh ^{2}(\rho)\left(\mathcal{L}_{\bar{u}} \bar{g}\right)(\bar{x}, \bar{y}) .
$$

In addition,

$$
\begin{aligned}
\left(\mathcal{L}_{f N} g\right)(x, y) & =g\left(D_{x} f N, y\right)+g\left(x, D_{y} f N\right)=d f(x) g(y, N) \\
& =d f(x) \sinh ^{2}(\rho) \bar{g}(N, y)=\sinh ^{2}(\rho)\left(\mathcal{L}_{f N} \bar{g}\right)(\bar{x}, \bar{y}),
\end{aligned}
$$

again because the same computation applies in $\mathbb{R}^{3}$. As a consequence, we have again that

$$
\left(\mathcal{L}_{v} g\right)(x, y)=\sinh ^{2}(\rho)\left(\mathcal{L}_{\bar{v}} \bar{g}\right)(\bar{x}, \bar{y})
$$

and (1) still holds. 
Corollary 1.4. Let $x, y \in S_{1,+}^{3}$ be two points such that the segment $s$ between $\bar{x}:=$ $\varphi_{S}(x)$ and $\bar{y}:=\varphi_{S}(y)$ intersects $B_{0}$. Let $v \in T_{x} S_{1}^{3}, w \in T_{y} S_{1}^{3}$ be two vectors, and let $\bar{v}:=\Phi_{S}(v), \bar{w}:=\Phi_{S}(w)$. Suppose that the first-order displacement of $\bar{x}$ and $\bar{y}$ by $\bar{v}$ and $\bar{w}$, respectively, does not change the Euclidean distance between $\bar{x}$ and $\bar{y}$. Then the first-order displacement of $x$ and $y$ by $v$ and $w$ does not change the hyperbolic length of $s$.

Recall that the hyperbolic length of $s$ was defined, before Theorem C, as the distance, in $H^{3}$, between the intersections of $s$ with the planes dual to $x$ and to $y$.

Proof of Corollary 1.4. Under the hypothesis of the corollary, there is an infinitesimal isometry $u$ of $\mathbb{R}^{3}$ (i.e. a Euclidean Killing vector field) such that $u(x)=v$ and $u(y)=w$. Let $\bar{u}:=\Phi_{S}(u)$, then $\bar{v}=\bar{u}(\bar{x}), \bar{w}=\bar{u}(\bar{y})$, and, under the deformation by $\bar{u}$, the distance between the planes dual to $\bar{x}$ and $\bar{y}$ does not change since, by Lemma 1.3, $\bar{u}$ is a Killing vector field.

Note that the proof actually uses only a weak version of Lemma 1.3, namely the fact that the Pogorelov map $\Phi_{S}$ sends de Sitter Killing vector fields to Euclidean Killing vector fields. This fact could presumably be proved more quickly by a direct computation, but it seems more illuminating to state the "real" property of $\Phi_{S}$.

Proof of Theorem B assuming Theorem $C$. Let $P$ be a polyhedron, suppose that there exists a closed ellipsoid $E$ containing no vertex of $P$ but intersecting all its edges. By replacing $E$ by a slightly larger ellipsoid if necessary, we can suppose that each edge of $P$ intersects $E$ along a segment.

We can also apply a projective transformation, so that $E$ is replaced by the unit ball $B_{0}$ in $\mathbb{R}^{3}$. This is possible because, by a result of Darboux [Da] and Sauer [Sau], infinitesimal rigidity is a projective property: given a projective transformation $u, P$ is infinitesimally rigid if and only if its image by $u$ is. Note that this result is not really necessary here, we could also work with an arbitrary ellipsoid $E$ and with the hyperbolic model given by its Hilbert metric (see, e.g., [Sc1]).

We can now consider $P$ as a hyperideal polyhedron $\bar{P}$ in the projective model. Let $v$ be a first-order deformation of $P$ which does not change its induced metric, and let $\bar{v}$ be the first-order deformation of $\bar{P}$ defined from $v$ by the Pogorelov map $\Phi_{S}$. By Corollary $1.4, \bar{v}$ (or rather its restriction to the vertices of $\bar{P}$ ) does not change, at first order, the edge lengths of $\bar{P}$. So, by Theorem C, $\bar{v}$ is a trivial deformation. So, by Lemma 1.3, $v$ is trivial. Therefore, $P$ is infinitesimally rigid.

\section{Hyperideal Simplices}

This section describes the Schläfli formula for hyperideal polyhedra, and some elementary properties of hyperideal simplices. Note that in this paper we only consider strictly hyperideal simplices and polyhedra; we sometimes refer to [Sc4], but the situation there was more complicated because it dealt also with polyhedra with some ideal vertices. 
Definition 2.1. The volume of a hyperideal simplex is defined as the volume of the compact polyhedron obtained by truncating each of the four ends by the plane dual to the corresponding hyperideal vertex.

The Schläfli formula describes the first-order variation of the volume of a polyhedron in a deformation, in terms of the first-order variation of its dihedral angles. We state it first for compact polyhedra. The proof can be found, e.g., in [Mi]. The dihedral angles mentioned here are the interior angles.

Lemma 2.2. Let $P$ be compact hyperbolic polyhedron, with edge lengths $\left(L_{i}\right)$ and dihedral angles $\left(\theta_{i}\right)$. In a deformation of $P$, the first-order variation of its volume is given by

$$
d V=-\frac{1}{2} \sum_{i} L_{i} d \theta_{i}
$$

Applying this formula to a truncated hyperideal polyhedron, we see that it holds also for hyperideal polyhedra, because the volume and edge lengths of a hyperideal polyhedron are the same as for its truncated polyhedron - and the angles at the truncation edges, which are equal to $\pi / 2$, do not vary. We thus obtain an extension of the Schläfli formula to hyperideal polyhedra (which has certainly been well known for some time).

Lemma 2.3. The Schläfli formula (2) is also valid for hyperideal polyhedra.

We now consider infinitesimal deformations of hyperideal polyhedra. Those deformations are uniquely determined by the first-order displacements of the vertices, basically one vector at each vertex.

Lemma 2.4. Let $S$ be a hyperideal simplex. There is no non-trivial first-order deformation of $S$ which does not change its edge lengths.

This statement is also well known, it can be proved directly in an elementary way. The reader can find such a proof in [Sc4]; we do not reproduce it here since it is not too surprising. The following lemma, concerning dihedral angles, is more subtle.

Lemma 2.5. Let $S$ be a hyperideal simplex. Its exterior dihedral angles are such that, for each vertex $s$ of $S$, the sum of the angles of the edges containing $s$ is greater than $2 \pi$, and equal to $2 \pi$ if and only ifs is ideal. Moreover, given a map $\alpha:\left\{e_{12}, \ldots, e_{34}\right\} \rightarrow(0, \pi)$ such that, for each vertex $s$ of $S_{0}$, the sum of the values of $\alpha$ on the edges of $S_{0}$ incident to $s$ is strictly larger than $2 \pi$, there exists a unique hyperideal simplex such that the exterior dihedral angle at each edge $e_{i j}$ is $\alpha\left(e_{i j}\right)$.

This is a simple special case of a recent description, by Bao and Bonahon [BB], of the possible dihedral angles of hyperideal polyhedra. Another proof of this result of Bao and Bonahon has been obtained by Rousset [Ro] as a consequence of the results of Rivin and Hodgson [RH] on compact hyperbolic polyhedra. 
The key point of the proof of Theorem $\mathrm{C}$ is the remark, made in the next lemma, that the volume of hyperideal simplices is a strictly concave function of the dihedral angles. It is taken, with its proof, from [Sc4]. The same is true for ideal polyhedra (see [Ri]), in that case it can be checked by a direct computation. It is however false for compact simplices. From here on we call $\mathcal{S}$ the space of hyperideal simplices (up to the isometries preserving the vertices). According to Lemma 2.5, $\mathcal{S}$ is the interior of a six-dimensional polytope.

Lemma 2.6. The volume of hyperideal simplices is a strictly concave function of the dihedral angles.

Proof. Let $S \in \mathcal{S}$ be a hyperideal simplex. Suppose that there is a direction in $T_{S} \mathcal{S}$ which is in the kernel of Hess $(V)$. Then by the Schläfli formula (2), the corresponding first-order variation of the edge lengths vanishes, and this is impossible by Lemma 2.5. Therefore, $\operatorname{Hess}(V)$ has a constant signature over each $\mathcal{S}$, with maximal rank. So it only remains to check that $\operatorname{Hess}(V)$ is negative definite at a point.

To do this one can consider a regular hyperideal simplex; by the Schläfli formula the question boils down to showing that the matrix of variations of the edge lengths with respect to the dihedral angles is positive definite. We refer the reader to [Sc4], where this is done (using a very short Maple program).

\section{Dihedral Angles of Hyperideal Polyhedra}

In this section we use ideas similar to those in previous papers dealing with dihedral angles of ideal polyhedra, in particular [Ri]. The situation is however simpler since, for hyperideal polyhedra, it is not necessary to choose horosphere around the vertices, etc. Of course, the fact that it is possible to extend those ideas from the ideal to the hyperideal context follows from Lemma 2.6.

Since we want to use the properties of the volume of simplices, we start by checking that our hyperideal (non-convex) polyhedron $P$ admits a non-degenerate triangulation, i.e. a decomposition in non-degenerate hyperideal simplices. We first remark that, in any cellulation of $P$ by hyperideal polyhedra $C_{1}, \ldots, C_{n}$, each vertex of the $C_{i}$ has to be a vertex of $P$.

Remark 3.1. Let $P$ be a hyperideal polyhedron. Suppose that the domain bounded by $P$ is the union of a finite number of convex hyperideal polyhedra $C_{1}, \ldots, C_{n}$, with disjoint interiors. Then the vertices of the $C_{i}$ are the vertices of $P$.

Proof. Suppose that some point $v$ is a vertex of at least one of the $C_{i}$, but is not a vertex of $P$. It is either in the interior of $P$, in the interior of a face of $P$ or in the interior of one of its edges. Moreover, by the definition of a hyperideal polyhedron, $v$ cannot be in $\overline{B_{0}}$.

Suppose that $v$ is in the interior of $P$. Then, since the $C_{i}$ are convex, at least one of the edges of the $C_{i}$ ending on $v$ is oriented in a direction which is not towards $B_{0}$. Thus some of the $C_{i}$ are not hyperideal polyhedra, a contradiction. 
Suppose now that $v$ is in the interior of a face $f$ of $P$. Let $b_{0}:=f \cap B_{0}$, then $v \notin \overline{b_{0}}$. The intersections of $f$ with the $C_{i}$ defines a cellulation of $f$, and the same argument as above shows that there is an edge of one of the $C_{i}$ which starts from $v$ in the direction "opposite" to $b_{0}$, and this again contradicts the fact that the $C_{i}$ are hyperideal.

Finally, the same argument works if $v$ is in the interior of an edge $e$ of $P$, since then $v$ separates $e$ into two parts, and only one of them can intersect $B_{0}$.

Definition 3.2. $\quad$ Let $P$ be a hyperideal polyhedron. A hyperideal triangulation of $P$ is a hyperideal cellulation by hyperideal polyhedra which are all simplices.

Lemma 3.3. Let $P$ be a hyperideal polyhedron. Suppose that $P$ has a hyperideal cellulation. Then it has a hyperideal triangulation.

Proof. We will show that it is possible to subdivide a hyperideal cellulation $C_{1}, \ldots, C_{n}$ to obtain a hyperideal triangulation. Note that we only have to prove that it is possible to do this affinely, because, given a convex hyperideal polyhedron $C_{i}$, any non-degenerate simplex with its vertices among the vertices of $C_{i}$ is a hyperideal simplex.

Let $v_{1}, \ldots, v_{v}$ be the vertices of $P$. For each $i \in\{1, \ldots, n\}$, we call $w_{i}$ the vertex of $C_{i}$ which has the smallest index. It is then clear that if $C_{i}$ and $C_{j}$ share a 2-face $f$, and if $w_{i} \in f$ and $w_{j} \in f$, then $w_{i}=w_{j}$.

For each $i \in\{1, \ldots, n\}$, we subdivide each of the 2-faces of the cellulation of $P$ which contains $w_{i}$ by adding the segments going from $w_{i}$ to all the other vertices of $f$. Since each 2-face contains at most one of the $w_{i}$, this leads to a triangulation of all the faces of the cellulation containing one of the $w_{i}$. We then further triangulate all the other non-triangular 2-faces of the cellulation in an arbitrary way.

Now we can subdivide each of the $C_{i}, 1 \leq i \leq n$, as follows: for each triangle $T$ of $\partial C_{i}$ which does not contain $w_{i}$, we add the simplex with one face equal to $T$ and the opposite vertex equal to $w_{i}$. Clearly, this defines a hyperideal triangulation of $P$.

Using Lemma 3.3, we can find a hyperideal triangulation $\tau$ of $P$. We stick to this triangulation until the end of the paper. We call $E$ and $N$ the number of edges and simplices in $\tau$, respectively.

Definition 3.4. We call $\mathcal{A}$ the space of possible dihedral angles for the simplices of $\tau$; $\mathcal{A}$ is the product of $N$ copies of the six-dimensional polytope determined by Lemma 2.5.

The polyhedron $P$, with its triangulation $\tau$, defines a set of dihedral angles on each of the simplices of $\tau$, i.e. an element of $\mathcal{A}$ which we call $\theta_{0}$. All the arguments that follow happen in the neighborhood of $\theta_{0}$.

An element $\theta \in \mathcal{A}$ determines, for each of the simplices of $\tau$, an identification with a unique hyperideal simplex. So each simplex of $\tau$ has a well-defined volume, and we can define the volume $V(\theta)$ as the sum of the volumes of the simplices of $\tau$. Since the sum of concave functions is concave, we see using Lemma 2.6 that:

Lemma 3.5. $\quad V$ is a strictly concave function on $\mathcal{A}$. 
Definition 3.6. We call $F: \mathcal{A} \rightarrow \mathbb{R}^{E}$ the map sending an element of $\mathcal{A}$ to the function defined, on each edge $e$ of $\tau$, by the sum of the (interior) dihedral angles at $e$ of the simplices of $\tau$ containing $e$. For each element $\alpha \in \mathbb{R}^{E}$, we call $\mathcal{A}(\alpha):=F^{-1}(\alpha)$.

Clearly, $F$ is an affine map, so that, for each $\alpha \in \mathbb{R}^{E}, \mathcal{A}(\alpha)$ is either empty or the intersection of $\mathcal{A}$ with an affine submanifold $\mathbb{R}^{E}$. We call $\alpha_{0}:=F\left(\theta_{0}\right)$.

Lemma 3.7. Let $\theta \in \mathcal{A}$, and let $\alpha:=F(\theta)$. $\theta$ is a critical point of the restriction of $V$ to $\mathcal{A}(\alpha)$ if and only if, for each edge e of $\tau$, the length assigned to e by all the simplices of $\tau$ containing it is the same.

Proof. Let $\dot{\theta}$ be a first-order deformation of $\theta$, in other words $\dot{\theta}$ gives a first-order deformation of each of the dihedral angles of each of the simplices $C_{1}, \ldots, C_{n}$ in the triangulation $\tau$ of $P$. By the Schläfli formula (2), the first-order variation of the volume under the deformation $\dot{\theta}$ is

$$
\dot{V}=-\frac{1}{2} \sum_{i=1}^{n} \sum_{j=1}^{e(i)} L_{i, j} \dot{\theta}_{i, j},
$$

where $e(i)$ is the number of edges of the polyhedron $C_{i}, L_{i, j}$ is the hyperbolic length of the edge $e_{j}$ of $C_{i}$, and $\dot{\theta}_{i, j}$ is the first-order variation, under $\dot{\theta}$, of the same edge.

This formula can also be written as

$$
\dot{V}=-\frac{1}{2} \sum_{i=1}^{e} \sum_{j=1}^{p(i)} \bar{L}_{i, j} \dot{\bar{\theta}}_{i, j},
$$

where now $e$ is the number of edges of the triangulation $\tau$ of $P$ (i.e. the sum of the number of interior edges and of the number of edges of $P$ ), $p(i)$ is the number of simplices among $C_{1}, \ldots, C_{n}$ which contain edge $e_{i}, \bar{L}_{i, j}$ is the hyperbolic length of edge $e_{i}$ for the $j$ th polyhedra containing it, and $\dot{\bar{\theta}}_{i, j}$ is the first-order variation of the dihedral angle of the same edge.

Suppose first that the length of each edge is the same for all the simplices containing it. In the notations of the previous formula, this means that, for each $i \in\{1, \ldots, e\}$, there exists a $\bar{L}_{i}$ such that, for each $j \in\{1, \ldots, p(i)\}, \bar{L}_{i, j}=\bar{L}_{i}$.

Then, with the same notations, we have for each $i \in\{1, \ldots, e\}$,

$$
\sum_{j=1}^{p(i)} \bar{L}_{i, j} \dot{\bar{\theta}}_{i, j}=\bar{L}_{i} \sum_{j=1}^{p(i)} \dot{\bar{\theta}}_{i, j}=0,
$$

which shows that, in this case, $\theta$ is a critical point of the restriction of $V$ to $\mathcal{A}(\alpha)$.

Conversely, suppose that there is an edge $e_{i_{0}}$ of the triangulation which is contained in two simplices, say $C_{j_{1}}$ and $C_{j_{2}}$, and that the hyperbolic lengths of the $e_{i_{0}}$ for $C_{j_{1}}$ and for $C_{j_{2}}$ are not the same. Consider the following first-order variation $\dot{\theta}$ of $\theta$ :

- The first-order variation of the angle at the edge $e_{i_{0}}$ of $C_{j_{1}}$ is equal to 1 .

- The first-order variation of the angle at the edge $e_{i_{0}}$ of $C_{j_{2}}$ is equal to -1 .

- The other angles remain the same. 
Clearly, the total angle at each edge of the triangulation remains the same, so that $\dot{\theta}$ is in the tangent space of $\mathcal{A}(\alpha)$. However, in (3), the term corresponding to the edge $e_{i_{0}}$ is not zero, while the sums for all the other edges vanish. So $\dot{V} \neq 0$, and $\theta$ is not a critical point of the restriction of $V$ to $\mathcal{A}(\alpha)$.

In particular, $\theta_{0}$ is a critical point of $V$ restricted to $\mathcal{A}\left(\alpha_{0}\right)$. Therefore, a direct consequence of Lemma 3.5 is:

Corollary 3.8. For each $\alpha \in \mathbb{R}^{E}$ close enough to $\alpha_{0}$, there is a unique $\theta_{c}(\alpha) \in \mathcal{A}(\alpha)$ which is a critical point of the restriction of $V$ to $\mathcal{A}(\alpha) . \theta_{c}(\alpha)$ depends smoothly on $\alpha$.

Proof. Since the $\mathcal{A}(\alpha)$, for $\alpha$ close to $\alpha_{0}$, define a foliation of a neighborhood of $\theta_{0}$ by affine subspaces, this is a direct consequence of the strict concavity of $V$.

Proof of Theorem D. Let $v$ be an infinitesimal deformation of $P$ which does not change its dihedral angles. We can find a one-parameter family $\left(P_{t}\right)_{t \in[0,1]}$ with $P_{0}=P$ and such that, for each vertex of $P$, the first-order displacement at $t=0$ is given by $v$.

The triangulation of $P$ obtained in Lemma 3.3 can be extended to a triangulation of $P_{t}$ for $t$ small enough, with the same combinatorics. Considering the dihedral angles of the simplices leads to a one-parameter family $\left(\theta_{t}\right)_{t \in[0, \varepsilon]}$ for some $\varepsilon>0$. By construction:

- For all $t \in[0, \varepsilon], \theta_{t}$ is a critical point of $V$ restricted to $\mathcal{A}\left(F\left(\theta_{t}\right)\right)$-this follows from Lemma 3.7 since our deformation comes from a deformation of $P$ among polyhedra.

- $\left(d F\left(\theta_{t}\right) / d t\right)_{t=0}=0$, since the dihedral angles of $P$ does not change at first order, and the total angles around the interior edges of the cellulation remain equal to $2 \pi$.

This clearly contradicts the strict concavity of $V$ unless $\left(d \theta_{t} / d t\right)_{t=0}=0$.

\section{Edge Lengths}

This section contains the proofs of Theorems $\mathrm{C}$ and $\mathrm{E}$. The basic idea is that once we know that polyhedra near $P$ are parametrized by their dihedral angles and that their volume is a strictly concave function of the dihedral angles, the Schläfli formula shows that the hyperbolic edge lengths - which appear again as the coefficients of the differential of $V$-have a non-zero first-order variation under any non-trivial deformation.

Definition 4.1. $\mathcal{P}$ is the space of hyperideal polyhedra with the same combinatorics as $P$. We still denote by $V$ the hyperbolic volume, seen as a function on $\mathcal{P}$.

We need the basic fact that the volume, as a function on $\mathcal{P}$, is a strictly concave function of the dihedral angles. It is a consequence of the following elementary remark, taken from [Sc2].

Remark 4.2. Let $\Omega \in \mathbb{R}^{N}$ be a convex subset, and let $f: \Omega \rightarrow \mathbb{R}$ be a smooth, strictly concave function. Let $\rho: \mathbb{R}^{N} \rightarrow \mathbb{R}^{p}$ be a linear map, with $p<N$, and let $\bar{\Omega}:=\rho(\Omega)$. 
Define a function:

$$
\begin{aligned}
\bar{f}: \bar{\Omega} & \rightarrow \mathbb{R} \\
y & \mapsto \max _{x \in \rho^{-1}(y)} f(x) .
\end{aligned}
$$

Then $\bar{\Omega}$ is convex, and $\bar{f}$ is a smooth, strictly concave function on $\bar{\Omega}$.

Proof. It is quite obvious that $\bar{\Omega}$ is convex, and also that $\bar{f}$ is smooth since $f$ is strictly concave.

Let $\bar{c}:[0,1] \rightarrow \bar{\Omega}$ be a geodesic segment, parametrized at constant speed. By definition of $\bar{f}$, there exist points $x_{0}, x_{1} \in \Omega$ such that

$$
\bar{c}(0)=\rho\left(x_{0}\right), \quad \bar{c}(1)=\rho\left(x_{1}\right), \quad \bar{f} \circ \bar{c}(0)=f\left(x_{0}\right), \quad \bar{f} \circ \bar{c}(1)=f\left(x_{1}\right) .
$$

Let $c:[0,1] \rightarrow \Omega$ be the geodesic segment parametrized at constant speed such that $c(0)=x_{0}$ and $c(1)=x_{1}$. Since $\rho$ is linear, $\bar{c}=\rho \circ c$.

Moreover, since $f$ is strictly concave

$$
\forall t \in(0,1), \quad f \circ c(t)>t f \circ c(0)+(1-t) f \circ c(1) .
$$

Therefore, the definition of $\bar{f}$ shows that

$\forall t \in(0,1), \quad \bar{f} \circ \bar{c}(t) \geq f \circ c(t)>t f \circ c(0)+(1-t) f \circ c(1)=\bar{f} \circ \bar{c}(0)+\bar{f} \circ \bar{c}(1)$.

This shows that $\bar{f}$ is strictly concave.

Proof of Theorem E. It follows directly from the previous remark (applied with $\Omega=$ $\mathcal{P}, f=V$ and $\rho=\alpha$ ) and from Lemmas 3.5 and 3.7. Note that this proves that $V$ is a strictly concave function on a larger deformation space, of which $\mathcal{P}$ is an affine subspace.

Proof of Theorem C. By the previous lemma, each non-trivial first-order deformation of $P$ induces a non-trivial first-order variation of the differential of $V$. By the Schläfli formula (2), the coefficients of $d V$ are the edge lengths, and the result follows.

Note that the proof actually shows a little bit more: the infinitesimal deformations of $P$ are locally parametrized by the variation of its edge lengths. Lemma 1.3 shows that the same result is also true for Euclidean polyhedra under the hypothesis of Theorem B.

\section{Acknowledgments}

I thank Francis Bonahon and Igor Rivin for some illuminating conversations related to this text, Victor Alexandrov, Bob Connelly and Idjad Sabitov for some very relevant remarks, and Greg McShane for the beautiful illustrations shown in Fig. 1. The text was also significantly improved thanks to helpful remarks from two anonymous referees. 


\section{References}

[An] E. M. Andreev. On convex polyhedra of finite volume in Lobacevskii space. Math. USSR-Sb., 12(3):225-259, 1971.

[BB] X. Bao and F. Bonahon. Hyperideal polyhedra in hyperbolic 3-space. Bull. Soc. Math. France, 130(3):457-491, 2002.

[Brä] W. Brägger. Kreispackungen und Triangulierungen. Enseign. Math. (2), 38(3-4):201-217, 1992.

[Bri] R. Bricard. Mémoire sur la théorie de l'octaèdre articulé. J. Math. Pures. Appl., Liouville, 3:113-148, 1897.

[Ca] A. L. Cauchy. Sur les polygones et polyèdres, second mémoire. J. Ecole Polytechnique, 19:87-98, 1813.

[CdV] Y. Colin de Verdière. Un principe variationnel pour les empilements de cercles. Invent. Math., 104(3):655-669, 1991.

[Co1] R. Connelly. A counterexample to the rigidity conjecture for polyhedra. Inst. Hautes Études Sci. Publ. Math., 47:333-338, 1977.

[Co2] R. Connelly. Rigidity. In Handbook of Convex Geometry, Vols. A, B, pages 223-271. North-Holland, Amsterdam, 1993.

[Da] G. Darboux. Leçons sur la théorie générale des surfaces. I, II. Éditions Jacques Gabay, Sceaux, 1993. Reprint of the second (1914) edition (I) and the second (1915) edition (II), Cours de Géométrie de la Faculté des Sciences. [Course on Geometry of the Faculty of Science.]

[De] M. Dehn. Über den Starrheit konvexer Polyeder. Math. Ann., 77:466-473, 1916.

[Le] A.-M. Legendre. Eléments de géométrie. Paris, 1793 (an II). Première édition, note XII, pp. 321-334.

[Mi] J. Milnor. The Schläfli differential equality. In Collected papers, Vol. 1. Publish or Perish, Houston, TX, 1994.

[Po] A. V. Pogorelov. Extrinsic Geometry of Convex Surfaces. Translations of Mathematical Monographs, Vol. 35. American Mathematical Society, Providence, RI, 1973.

[RH] I. Rivin and C. D. Hodgson. A characterization of compact convex polyhedra in hyperbolic 3-space. Invent. Math., 111:77-111, 1993.

[Ri] I. Rivin. Euclidean structures on simplicial surfaces and hyperbolic volume. Ann. of Math., 139:553580, 1994.

[Ro] M. Rousset. Sur la rigidité de polyèdres hyperboliques en dimension 3: cas de volume fini, cas hyperidéal, cas fuchsien. math.GT/0211280; Bull. Soc. Math. France, 132:233-261, 2004.

[RR] L. Rodríguez and H. Rosenberg. Rigidity of certain polyhedra in R3. Comment. Math. Helv., 75(3):478$503,2000$.

[Sab] I. Kh. Sabitov. The volume of a polyhedron as a function of its metric. Fund. Prikl. Mat., 2(4):12351246, 1996.

[Sau] R. Sauer. Infinitesimale Verbiegungen zueinander projektiver Fläschen. Math. Ann, 111:71-82, 1935.

[Sc1] J.-M. Schlenker. Métriques sur les polyèdres hyperboliques convexes. J. Differential Geom., 48(2):323405, 1998.

[Sc2] J.-M. Schlenker. Hyperbolic manifolds with polyhedral boundary. Preprint math.GT/0111136, available at http://picard.ups-tlse.fr/\%schlenker, 2001.

[Sc3] J.-M. Schlenker. Hyperbolic manifolds with convex boundary. Preprint math.DG/0205305, available at http://picard.ups-tlse.fr/\%schlenker, 2002.

[Sc4] J.-M. Schlenker. Hyperideal polyhedra in hyperbolic manifolds. Preprint math.GT/0212355, 2002.

[St] J. J. Stoker. Geometrical problems concerning polyhedra in the large. Comm. Pure Appl. Math., 21:119-168, 1968.

[Th] W. P. Thurston. Three-dimensional geometry and topology. Recent version of the 1980 notes. http://www.msri.org/publications/books/gt3m/, 1997.

Received May 13, 2003, and in revised form November 17, 2003. Online publication June 7, 2004. 